\section{Hunting the helix}

How does a DNA-binding protein

recognize its alloted site? If it can 'feel' inside the helical grooves it will encounter the edges of the bases; but the helix geometry may itself vary with sequence. G. G. Privé et al. (J. molec. Biol. 217, 177-199; 1991) compare the structures of three crystallographically isomorphous decanucleotides in the form of self-complementary B-DNAs. The width of the minor groove and other features (including hydration pattern) vary markedly with composition. Crystal packing perturbs the structure at some sequence conjunctions, which therefore define the intrinsically 'soft', distortionprone parts of a helix. In a following report by the same group, K. Yanagi et al. (201-214) draw together the 11 available high-resolution structures and look for empirical rules. A general predictive algorithm is not yet within reach, as the structure depends not only on nearest neighbours but also on the base pairs on either side: of the 136 four-base-pair combinations only 33 are represented in the known structures.

\section{Gut reactions}

SMOKERS who are also drinkers will find cause for rumination in a report from an Australian group published in the British Medical Journal (302, 20-23; 1991) as will traffic police. R. D. Johnson and colleagues find that cigarette smoking delays the absorption of alcohol into the bloodstream. This is a result of the slowing of passage of the stomach contents into the small intestine, the principal site of alcohol absorption. The authors point out that the finding has implications for legal proceedings in suspected cases of drunken driving, because the back-calculation of blood alcohol levels to the time of a vehicle accident is more complicated than it might have seemed.

\section{Bright ideas}

A TEAM at the Technische Hochschule Darmstadt has devised a bright $X$-ray source, tunable from 5 to $20 \mathrm{keV}$, using the remarkable phenomenon of channelling radiation. The physical principle exploited by $\mathrm{H}$. Genz et al. (Appl. Phys. Lett. 57, 2956-2958; $1990)$ is the radiation of photons by high-energy electrons passing along a chain of atoms in a perfect crystal: varying the electron energy tunes the $X$-rays. Although the $X$-ray intensities obtained so far are lower than those from conventional synchrotron sources, the new device relies only on an electron accelerator a few metres long (and a small crystal), not the vast storage rings used at the dedicated laboratories such as at Daresbury in the UK and Grenoble in France. As a result, similar systems could easily be deployed in university or hospital laboratories.

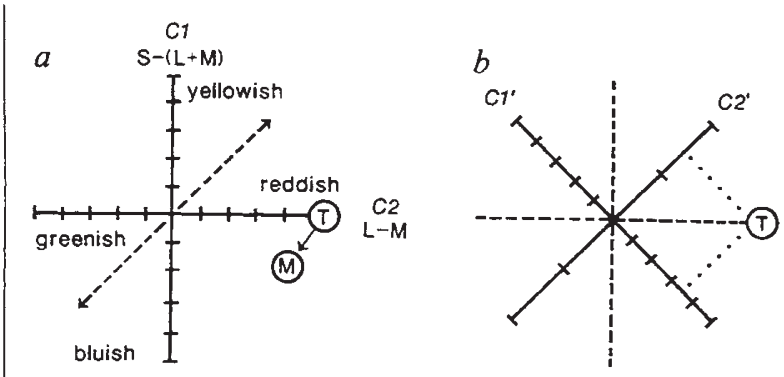

a, A constant-luminance plane in colour space $(L+M=$ constant). The axes $C 1$ and $C 2$ are aligned along the cardinal axes: along $C 1(S-(L+M))$, colours vary from greenish-yellow to reddish-blue; along $C 2(L-M)$, colours vary from reddish to greenish. After an observer adapts to a field varying between orange and turquoise along the dotted line, the hue of a test light on axis $C 2(T)$ is shifted in the bluish direction $(\mathrm{M})$. $b$, This could result from a realignment of the axes in the adapted field. In the new coordinate system, the test light stimulates both $C 1^{\prime}$ and $C 2^{\prime}$, and the mechanism underlying $C 2^{\prime}$ is desensitized. To match $T, M$ must therefore have components along both $C 1$ and $C 2$. (The predicted hue shift is larger than observed: It may be that the axes are realigned all across the visual field, but the sensitivity of $C 2^{\prime}$ is diminished only in the adapting field.)

orthogonal directions in colour space (for example red-green and purple-yellow) the patterns do not cohere but slip against each other. If the colour variations are along intermediate directions, they again cohere. It seems that colours are analysed into cardinal components before they contribute to motion perception.

But might there be yet a third stage of colour encoding? If the perception of chartreuse is represented at the second stage by a combination of activities in the $\mathrm{L}-\mathrm{M}$ and $\mathrm{S}-(\mathrm{L}+\mathrm{M})$ opponent mechanisms, then there could be a higher-order mechanism that lights up when it registers that combination. If so, there should be many such mechanisms, and the colour code at that level would consist of a large array of their activities.

Using a technique similar to that of Krauskopf et al. ${ }^{+}$, Webster and Mollon find surprisingly different results. These imply that such a third stage does exist. Instead of measuring the detectability of faint test flashes, Webster and Mollon asked observers to match the colour of supra-threshold test lights after adapting to temporally modulating fields. As one would predict from the results of Krauskopf et al., when the observers adapted to colour variations along a cardinal direction, the greatest change in colour appearance occurred for test lights along that direction and the least change for test lights along the orthogonal direction. But for some observers, this was also true for intermediate adapting directions: for every adapting direction tried, the greatest change was in that direction, the least in the orthogonal direction. Krauskopf et al. found hints of a similar effect in their original study defining the cardinal directions: for some observers, some selective desensitization also occurred for oblique directions. Thus there seem to be many mechanisms involved in encoding colour, each tuned to a distinct direction in colour space and each independently susceptible to desensitization.

This technique relies on the time-honoured tenet of psychophysics, that adaptation reveals selective mechanisms by fatiguing them. But many psychophysicists are themselves weary of fatigue as the explanation for adaptation. Barlow and Foldiak ${ }^{8}$ argue that the term fatigue obscures the natural purpose of desensitization: the visual system may deliberately reduce its sensitivity to frequently occurring combinations or 'contingencies' as a way of storing information about the natural world. This enables it to be more sensitive to the unexpected.

What contingencies might be stored for colour? One possibility is suggested by Lee's observation" that the locus of daylight chromaticities forms a straight line in the constant-intensity plane of cone responses, and that this line falls close to the blue-yellow axis. So the visual system seems to have adapted to the natural variations in light it encounters by aligning one of its colour-coding axes along the same direction, and the others in the orthogonal directions.

This argument leads to another interpretation of Webster and Mollon's results. First note that they are not exactly what one would expect from truly independent mechanisms. If each discriminable direction in colour space were represented by a strictly independent mechanism, then variations along one direction would not affect the activity of mechanisms tuned to other directions. Test stimuli placed anywhere except on the adapting axis should therefore appear unchanged, not just those placed on the orthogonal axis.

The results are perhaps more consistent

1. Webster, M. \& Mollon, J. Nature 349, 235-238 (1991).

2. Buchsbaum, G. \& Gottschalk, A. Proc. R. Soc. B220, $89-113(1983)$

3. Jameson, D. \& Hurvich, L. J. opt. Soc. Am. 45, 546-552 (1955).

4. Krauskopf, J., Williams, D.R. \& Heeley, D.W. Vision Res. 22, $1121-1131$ (1982)

5. MacLeod, D.I.A. \& Boynton, R.M. J. opt. Soc. Am. 69 1183-1186 (1979).

6. Flanagan, P., Cavanagh, P. \& Favreau, O.E. Vision Res. 30, $769-778$ (1990).

Krauskopf, J. \& Farell, B. Nature 348, 328-331 (1990).

8. Barlow, H. \& Foldiak, P. in The Computing Neuron (eds Durbin, R.M., Miall, C. \& Mitchison, G.J.) $54-72$ (Addison-Wesley, Wokingham, 1989).

9. Lee, H.-C. Eastman Kodak Tech. Rep. (Rochester, New York, 1989).

10. Derrington, A.M., Krauskopf, J. \& Lennie, P. J. Physiol. 357, 241-265 (1984).

11. Ts'o, D.Y. \& Gilbert, C.D. J. Neurosci. 8, 1712-1727 (1988).

12. Desimone, R., Schein, S.J., Moran, J. \& Ungerleider, L.G. Vision Res. 25, 441-452 (1985). 